\title{
En Torno a la Filosofía de la Educación em Panamá
}

\author{
REYES, Abdiel Rodríguez
}

\section{RESUMO}

Neste artigo, abordaremos alguns pontos relacionados à Filosofia da Educação (FE) no Panamá. Primeiro, vamos problematizar o assunto do que é nossa concepção de educação no Panamá; em segundo lugar, procuramos destacar a figura de Diego Domínguez Caballero como promotor da FÉ propriamente; Por último, analisaremos o conteúdo do curso FE como tal. Estes três pontos nos darão uma primeira aproximação ao que é a FE no Panamá. Os pontos serão desenvolvidos na mesma ordem exposta. Outro elemento que não vamos ignorar ao longo deste trabalho são os problemas de natureza mais geral em relação à sociedade em que estão inseridos os três pontos que analisaremos e que consideramos interceptar. Esses problemas serão tratados em linhas gerais, pois, em suma, é um tópico consubstancial à FE.

Filosofia. Educação. Panamá. Diego Dominguez Cabellero.

\section{Around Philosophy of Education in Panama}

\section{ABSTRACT}

This article approaches some points about Philosophy of Education in Panama. Firstly, we problematize the subject of what is that we call our concept of education in Panama; Secondly, we put our effort into standing out the persona of Diego Domínguez Caballero as a Philosophy of Education promoter thoroughly. Lastly, we analyse the content of the Philosophy of Education course, as it denotes. These three steps will give us a first approximation with what the Philosophy of Education in Panama is. The points will be developed in the same order they were exposed. Some other elements we won't ignore along this piece of writing are the problems, in general, related to the society where these three points, which we'll analyse and regard intercepting, are inserted. These problems will be dealt with, generally speaking, because, in short, they are one consubstantial topic to the Philosophy of Education.

\footnotetext{
1 Profesor de Filosofía e investigador en el Centro de Investigaciones de la Facultad de Humanidades de la Universidad de Panamá. Presidente de la Asociación Centroamericana de Filosofía. Correo: abdiel.rodriguezreyes@up.ac.pa
} 
Philosophy. Education. Panama. Diego Dominguez Caballero.

\title{
A MODO DE INTRODUCCIÓN
}

\author{
Es axiomático que la educación que se imparte en los \\ centros de estudio debe correr pareja con las \\ transformaciones de índole material y moral que tienden a \\ mejorar las condiciones de vida en el país. \\ Rafael E. Moscote
}

En este artículo abordaremos algunos puntos relacionados a la Filosofía de la Educación (FE) en Panamá. Primero, problematizaremos la temática de cuál es nuestra concepción de la educación en Panamá; segundo, buscamos resaltar la figura de Diego Domínguez Caballero como promotor de la FE propiamente; por último, analizaremos el contenido del curso de la FE como tal. Estos tres puntos nos darán una primera aproximación a lo que es la FE en Panamá. Éstos se desarrollarán en el mismo orden expuesto. Otro elemento que no pasaremos por alto a lo largo de este trabajo son los problemas de índole más general concernientes a la sociedad en la que están insertos los tres puntos que analizaremos y que consideramos que se interseccionan. Dichos problemas serán tratados a grandes rasgos entre líneas ya que, en definitiva, es un tema consustancial a la FE.

Domínguez Caballero fue quien más promovió la FE en Panamá, como docente y planificador, de tal forma que, no puede pensarse el tema sin tomarlo en cuenta. Por eso, para aproximarnos a lo que es la FE en Panamá tomaremos en cuenta su obra, lo cual nos ubica en la mitad del siglo XX cuando empieza a germinarse la FE. Una simple revisión bibliográfica confirmaría que fue Domínguez Caballero de los pocos que escribió explícitamente sobre la temática, además de defender los espacios de FE en los programas de educación superior en la Universidad de Panamá e, incluso, en la educación superior en general. Su principal argumento buscaba mantener como telón de la educación la "meditación filosófica" que hoy cuenta con poco espacio. Fue un ferviente defensor de la filosofía en general y, en particular, de la FE aunque, al final, no logró del todo su cometido, en la actualidad hay una pedagogización de la educación superior; todo parece indiciar que la educación superior, en general, se concibe como pedagógica-escolarizada prioritariamente. La Universidad de Panamá no escapa de esa realidad, a pesar de los intentos, no logra conjugar la investigación científica con la enseñanza.

El contexto histórico desde donde podemos hablar, propiamente, de la FE en Panamá es el período republicano y, en específico, a partir de la fundación de 
la Universidad de Panamá. En los primeros años de la república, fue una pléyade de pensadores liberales, mayoritariamente, los que concibieron toda una filosofía de la educación en diversos niveles, desde la escuela primaria hasta los estudios superiores. Es decir, tenían toda una concepción filosófica de la educación que se erigía con la reciente república pese a todos los tropiezos y la troquelación en 1903. Con esas limitaciones, el país no podía andar a ciegas, tenía que escolarizarse y, además, formarse un sentimiento nacional, tarea que cumplió de alguna u otra forma el Instituto Nacional y la Universidad Nacional en el siglo pasado. Se forjó un espíritu crítico, lo que Octavio Méndez Pereira llamaría "la conciencia crítica de la nación" que es una especie de llama que necesita de vestales para que no se extinga el fuego y se pueda iluminar el camino aún por recorrer.

En lo que respecta a la Universidad de Panamá, en los primeros años fueron profesores extranjeros quienes ocuparon las primeras cátedras y formaron a los nacionales, en su mayoría, procedían de universidades alemanas: fueron aquellos que emigraron por el acecho nazi. Hay que tener en cuenta la rigurosidad de la academia alemana que permeó a esa primera generación en la que estuvo Domínguez Caballero. Él perteneció a la primera generación de egresados de filosofía, diríamos que se trató del proceso de profesionalización de la filosofía en Panamá, es decir: contar graduados de formacióon universitaria en la carrera de filosofía, aunque claro está que la filosofía no necesita de ningún título para ejercerla. A lo largo de nuestra historia hay muchas manifestaciones filosóficas que podemos detectar desde las cosmologías de los pueblos aurorales. Cuando hay amor a la sabiduría, sin ser profesionales en sentido estricto, hay filosofía; la curiosidad por el mundo en que se vive siempre ha acompañado al ser humano en su evolución. Nos circunscribimos a lo profesional, sólo para delimitar nuestro objeto de estudio que será la FE en Panamá. Antes de la profesionalización de la filosofía en Panamá, contamos con una rica tradición de pensamiento liberal, donde se erige lo bueno, lo malo y lo feo de lo que nos queda de institucionalidad en términos políticos, culturales y educativos. Somos deudores de ese liberalismo que se ha descompuesto por el propio deterioro de la clase política.

En la actualidad estamos, como gran parte del mundo, bajo el acecho del neoliberalismo en el que la filosofía puede pasar al descarte de los planes y programas de educación tanto a nivel escolar como universitario. Hay toda una concepción neoliberal de la Universidad criticada por pensadores como Noam Chomsky, Boaventura de Sousa Santos y Martha Nussbaum, entre los más citados. La mercantilización del conocimiento ya es evidente en Panamá, la proliferación de universidades garajes es una evidencia empírica. Simultáneamente, la falta de mística generalizada empeora aún más el estado de 
la cuestión. Estamos en un laberinto sin tener el hilo de Ariadna, por lo tanto, nos podemos extraviar.

Domínguez Caballero defendió los espacios para la filosofía, en general, y de la educación, en particular, en el sistema educativo panameño, visualizó el problema de la pedagogización-escolarización de la educación superior en el que nos encontramos actualmente. Hay que hacer notar que lo pedagógico y lo escolar es necesario, pero resulta poco enriquecedor mantenerse únicamente bajo ese esquema, es necesario trascenderlo. Para eso es útil la filosofía en sentido estricto. Lo escolar es la enseñanza obligatoria que tenemos por adquirir y lo pedagógico es enseñar con claridad los diversos contenidos. No debemos sobreponer esto sobre la filosofía, que tendría la excelsa tarea de organizadora y orientadora del conocimiento, la investigación, la erudición y el vinculo entre el obrar humano. En términos occidentales, para eso es la universidad.

La Universidad de Panamá debería ser puntera en la investigación y producción de conocimiento para el desarrollo y con impacto nacional, e incluso, internacional. Pero la realidad es otra, se está con la permanente tensión de cómo encarar las falencias filosóficas y pedagógicas. La intencionalidad tiene que traducirse en factibilidad para lograr los cambios necesarios y salir de esa obnubilación que a veces no nos permite ver la centralidad de la investigación. A pesar de las limitaciones esa intencionalidad no se pierde del todo.

Para que una universidad sea tal, por lo menos debe investigar, las universidades garaje procuran, en el mejor de los casos, aparentar hacerlo. Los indicadores que tienen que ver con la investigación se cubren mediocremente sólo para cumplir con las evaluaciones mínimas del Consejo Nacional de Evaluación y Acreditación Universitaria de Panamá (CONEAUPA). La proliferación de universidades garajes que comercian títulos que sólo tienen valides en el ámbito nacional son una caricatura del papel que debe jugar la universidad en la sociedad. Una muestra de la validez de un título de una universidad garaje es que, las universidades en el extranjero tienen como requisito que los títulos extranjeros cuenten con cierta cantidad de créditos y horas de estudio, en su mayoría cuatro años, muchas de las universidades garajes no cumplen siquiera este requerimiento, por lo tanto, no serían aceptados en ninguna universidad seria en el mundo. Tienen poca o nula investigación. No producen ni un panfleto más que el de la promoción de sus títulos.

Para culminar con este tema, son varios los problemas sin potencialidad de resolverse. Por señalar alguno de los más complejos, podemos hablar de la carencia de un sistema nacional de investigación real, de incentivos y de promoción de la investigación, como si lo tienen, por ejemplo: Argentina (CONICET) y México (CONACYT). La diferencia entre contar con un sistema de 
investigación -o no - se refleja en la asimetría de producción de conocimiento. Hay una diferencia porcentual abismal en la producción de conocimiento. En definitiva, será un problema mayúsculo que Panamá deberá encarar en los años próximos.

Domínguez Caballero hablaba de la "actividad de ardilla" para referirse a los esfuerzos aislados, sin un esprit de corps, de encontrar la unidad en al menos un punto estratégico, por ejemplo: de defensa de la filosofía, cualitativa y cuantitativamente a partir de la heterogeneidad de tradiciones y perspectivas. A pesar de que Diego Domínguez fuese conservador, en otros términos, en lo relativo a la defensa de los espacios de la filosofía de la educación, fue un férreo y organizado militante. Como sabrán, las ardillas se mueven y, al final, parecen no estar en ninguna parte, así mismo ocurre a la hora de organizar una defensa de la filosofía en Panamá. Así pasó con la defensa de la FE que al final perdió espacios significativos, en su lugar quedó la pedagogía y otro curso que se le asignó recientemente a la filosofía: la Teoría de la Ciencia.

La FE, grosso modo, también trata sobre la forma en cómo concebimos la educación: escolar y universitaria, que, como hemos dicho, abordaremos entre líneas. Incluso, habría que hurgar en las raíces ontológicas, como lo hicieron en su momento varios que presentaron sus trabajos de grado y reflexiones posteriores. En esa dirección, podemos mencionar a: Ricaurte Soler, Ricardo Arias Calderón, Isaías García Aponte, Moisés Chong y Edilia Camargo. Cuestión en donde pioneramente ahondó Domínguez Caballero; e incluso, dirigió algunos de esos trabajos, pero no abordaremos lo ontológico, dado que estaremos más enfocados en la FE en los términos antes expuestos.

\section{Temática}

La filosofía propiamente "profesional" se desarrolló en la Universidad de Panamá. Tan sólo tiene un poco más de ochenta años. La Universidad de Panamá, en comparación con otras universidades de la región, es un centro de educación superior lozano que aún tiene, pese a sus deficiencias, mucho que brindarle a la sociedad, y debe encontrar ese enganche que permita su vinculación concreta. En principio, tendría que producir un conocimiento emancipador y formar ciudadanos responsables con un futuro sostenible. Allí la gran tarea en donde la filosofía puede aportar en sus recovecos más abstrusos. En ese sentido, hay que tener una concepción filosófica de la educación, para que el sistema de educación en general tenga contenido. 
Para aproximarnos a la temática, rondaremos lo relativo a nuestro tema central: la FE en Panamá. Como decíamos, la filosofía de la educación encuentra su ágora en la Universidad de Panamá. A partir de 1903, con el nacimiento de la República, inmediatamente las elites liberales echaron a andar la rueda de la fortuna del reciente "país", para lograrlo necesitaban, como en todas las experiencias en otras latitudes, uno de los ejes medulares: la educación pública. Por un lado, para preparar a la mano de obra técnica e intelectual para las funciones públicas que se requerían, pero, por otro lado, e incluso de mayor peso espiritual, la forja de la "identidad nacional". Ese papel lo jugó el Instituto Nacional y luego la Universidad de Panamá. En los momentos más álgidos de nuestra historia republicana ambas instituciones han sido decisivas en pos de salvaguardar lo limitado de nuestra soberanía en el siglo XX.

El ideal universitario que deberíamos acuerpar tendría que ser aquella máxima kantiana de isapere aude! (atrévete a pensar) a partir del propio conocimiento, sin la tutela de otro. En los inicios del siglo XX, esa élite intelectual liberal al ver las necesidades que surgían con el novo "país," emprendieron la idea de tener una universidad, en principio elitista, pero que al pasar de los años tomó un carácter popular que cobró mayor fuerza con el proceso torrijista y que se mantiene pese a sus dificultades y limitaciones hasta hoy. En las tres primeras décadas del siglo xx la idea empezó a tomar fuerza.

Para tener una idea de cómo se concretó la Universidad en Panamá, véase la sugerente obra de Cesar del Vasto que registra desde Castilla de oro hasta la celebración de los setenta y cinco años de la Universidad de Panamá, pasa por la Universidad del Istmo de 1841, entre otros intentos. En cada uno de estos conatos se enseñó filosofía, en la Universidad colonial, fundamentalmente, bajo los principios escolásticos propios de la época. En estos menos de doscientos años, fluyeron ideas filosóficas que, muchas veces, no fueron el fiel reflejo de la realidad o, para decirlo en otros términos, no había un sentido de pertenencia.

Como es de amplio conocimiento, en 1903 Panamá se separa de Colombia bajo el amparo de Estados Unidos, el cual impuso un enclave colonial en donde los estadounidenses tenían una colonia dentro de Panamá, con sus propias leyes e instituciones públicas. El país se modificó considerablemente, resultado de la organización del territorio y la formación de lo que se llamaría la quinta frontera. En el ínterin de la fundación de la Universidad de Panamá en la década del treinta, diversas manifestaciones de pensamiento filosófico que pronto se aglutinarían en los claustros universitarios, evolucionaron en el marco de la naciente república. En primer lugar, el Instituto Nacional jugaría el papel de brindar educación para cubrir la demanda de profesionales. El Instituto Nacional es un baluarte espiritual del país por ese papel de formador de profesionales y, además, en la forja de una 
conciencia crítica en las luchas nacionalistas por la recuperación de la zona del Canal. Mucho de lo que sería una filosofía de toda la educación en Panamá proviene de allí, de esa visión de conjunto que se desarrolló en ese entorno y contexto; de lo más excelso a lo más revolucionario. La lista de prometedores profesores y políticos graduados de allí sería infinita; por lo que consideramos decisivo el papel que jugó el Instituto Nacional en buscarle un sentido a la educación en Panamá.

Uno de los intentos por establecer un claustro de educación superior fue la de Genarina de la Guardia en 1912. La iniciativa que sería financiada por fondos filantrópicos tenía que pasar por la aprobación de los Estados Unidos por nuestra condición de tutelaje, así que se tuvo que pasar por un largo proceso que, al final, no se concretó, este intento que buscó llamarse la Universidad Panamericana de Panamá fracasó. Se tuvo que esperar algunos años más para que

El 6 de mayo de 1918, la Facultad Nacional de Derecho y la Escuela de Derecho inician sus operaciones académicas con una matrícula de 130 y 72 estudiantes respectivamente. Pablo Arosemena, Santiago de la Guardia, Horacio y Ricardo J. Alfaro, José D. Moscote, Harmodio Arias Madrid, Samuel Quintero, Julio J. Fábrega, Daniel Chanis, Eusebio A. Morales, Dámaso A. Cervera, Gil R. Ponce, Humberto Tejera, Eduardo Chiari, Ricardo A. Morales, Federico Calvo y, finalmente, L. Carrol, profesor invitado de la Universidad de Barcelona, España, forman parte del cuerpo docente. (DEL VASTO, 2010, p.27).

Si tomamos en consideración estos nombres nos daremos cuenta de que son los referentes en donde se asientan la educación y artes liberales en Panamá, todos con brío en sus ámbitos de saber, colaboraron en la fundación del ideal universitario que se mantiene hasta hoy. Otros nombres prestantes se vincularon a este proyecto, como Belisario Porras, Jeptha B. Duncan, Daniel Crespo, Temístocles R. Céspedes, Otilia Arosemena de Tejeira y Octavio Méndez Pereira. Éste último será el arquitecto de la Universidad de Panamá que se fundó posteriormente. Cabría decir que, no se puede hacer nación sin universidad, sería una contradicción en los términos. Estos primeros pasos en materia educativa, en el período republicano, serían los proto momentos de la FE en Panamá, que siguieron el canon de la universidad occidental.

Después del intento de Genarina de la Guardia; otro intento por establecer un centro de educación superior fue el de Acción Comunal en la primera mitad de la década del treinta, pero tampoco cuajó del todo. Fue hasta 1935 que por fin se pudo consolidar el proyecto de una Universidad Nacional de Panamá, pero es hasta 1946 que adoptó el nombre que se mantiene hasta hoy: Universidad de Panamá. 
El 20 de diciembre de 1946, gracias a las gestiones del Ministro de Educación, José Daniel Crespo, respaldado por los docentes Abel Bravo, Ricardo J. Alfaro y Octavio Méndez Pereira, se aprueba la Ley 40 , por medio de la cual se traspasan terrenos estatales ubicados en El Cangrejo y otros lugares a la Universidad Nacional de Panamá. El 24 de diciembre de 1946 también se aprueba la Ley Orgánica de Educación (Ley 47), que regula el funcionamiento de la institución que, a partir de ese momento se denominará Universidad de Panamá. (DEL VASTO, 2010, p.41).

Es una universidad joven que, en su corta edad, es la que ha formado a la mayoría de los profesionales del país, incluidas a las profesiones liberales, que es donde nos queremos detener; la Universidad de Panamá durante toda la segunda mitad del siglo $x x$ formó en humanidades y letras, fue el único espacio donde se enseña filosofía, propiamente, en el Departamento de Filosofía como el único espacio institucional y académico en el país que forma profesionales en la materia en sus dos escuelas: la de Filosofía, Ética y Valores; y Filosofía e Historia. Con la limitación, no sólo del Departamento, sino de la propia Universidad como tal, de concebirse como un espacio pedagógico-escolarizado en el sentido de carecer de un sistema de investigación eficiente, esto afecta directamente el estatus de la universidad en los rankings internacionales.

Sobre otros tópicos que tienen que ver con la temática, tenemos que, por lo menos, mencionar otros antecedentes sobre quiénes fueron los pensadores que concibieron el sistema de educación nacional, aunque con una gran influencia norteamericana y europea. En el prólogo de una selección de textos de José Dolores Moscote de la Biblioteca de la Nacionalidad, Pedro Pineda González, señaló que a: "José Dolores Moscote le correspondió indicar y determinar las nuevas formas ideológicas del Estado panameño [...] sintieron la necesidad de proveer al nuevo Estado de elementos jurídicos y educativos por la misma forma inédita como éste surge" (Cf. DOLORES MOSCOTE, 1999, p.3). Desde aquí, podemos englobar la concepción, en su amplitud, de lo que sería la FE en Panamá.

La estructura de nuestro sistema educativo estuvo bajo la egida de los liberales que, como hemos visto, se ha descompuesto y ha quedado en mano de los neoliberales. Por eso es notoria la poca inversión pública en artes y humanidades, con lo que se deja al Estado sin herramientas con las cuales encarar la deshumanización mundial. Eso explica, entre otras cosas, la falta de sensibilidad, el desaliño social y el aumento de la delincuencia. Hay un evidente proceso de desmontaje de la importancia de la educación como elemento emancipador y de movilidad social. 
Entre otros aspectos, en la concepción que se formó en Panamá, ya desde mediados del siglo XX. Alfredo Cantón se planteaba hace sesenta años un problema aún sin resolver, el de la educación en el interior del país. Los que han tenido que ver con la educación, al menos en los períodos post invasión, no han planificado en función del desarrollo necesario en las comunidades rurales del país. Panamá es un país "canalero", es decir, que gira en torno al canal. En vista de eso, en una actitud supina, la burguesía rentista enfocó todos sus intereses a esa dinámica, sin explorar otros campos. Por ejemplo, en un sector agropecuario en crisis que se encuentra sin una política enfocada al desarrollo de ese sector.

Para la década del cincuenta, Alfredo Cantón (1952) ya tenía toda una concepción de cómo debería ser la escuela rural en Panamá. Entre los puntos que planteó están: despertar un interés por la naturaleza; formar una actitud científica en el niño; la integridad moral en la formación de ciudadanía y, por último; formar a un ciudadano preocupado por su comunidad. Sesenta años después, estos problemas siguen sin resolverse. Por no tener una mirada de largo alcance y no tomar en cuenta la tradición, las preocupaciones educativas se han acumulado desde los inicios republicanos. Hoy, el país tiene un abismo entre el servicio que presta en las áreas rurales de difícil acceso con respecto a las de la ciudad capital. Incluso, los antagonismos se reflejan en diversas actitudes territoriales; de igual forma, como hay dos Panamá, también hay dos sistemas educativos, sin entrar en las diferencias internas.

En la actualidad, los problemas urgentes son de índole administrativa. Contradictoriamente, somos de los países con el crecimiento económicamente más alto de la región, sin embargo, aún no invertimos el $6 \%$ del PIB en educación. Por eso, vemos la precariedad de mucha de la infraestructura de las escuelas y colegios, la falta de equipo tecnológico, entre otras deficiencias. Lo anterior, aunado también a la ineficiencia del órgano legislativo: en ocasiones no se invierte todo el presupuesto o no se corresponde con las necesidades materiales de la comunidad económica, y a nivel más operativo, los profesores recién nombrados demoran meses para recibir su salario, lo cual es inconcebible.

De vuelta sobre la temática, además de los aspectos señalados, hay un análisis dicotómico interesante que hace Diógenes de la Rosa; una de las mentes más conspicuas de Panamá. Antes de proseguir con lo que plantea de la Rosa, quiero rescatar lo que dice el pensador colombiano Carlos B. Gutiérrez al plantear el re-pensar aquel ideal renacentista de que las humanidades y la lectura de los clásicos sirvieran de acicate para hacer del ser humano, más humano. Es precisamente sobre este ideal que De la Rosa rodea. También se despoja de ese ropaje eurocéntrico de la supremacía de Grecia y Roma e indica que hay humanismo "en todas las culturas históricamente conocidas". Concluye que: "la 
educación debe ser humanista" (DE LA ROSA, 1985, p.393) en cuanto sensibilizar a la sociedad, cuestión que hemos perdido en nuestro sistema de educación.

\section{Diego Domínguez Caballero y la Filosofía de la educación}

Diego Domínguez Caballero fue el eje central de la profesionalización de la filosofía en Panamá, fue el primer estudiante y el primer profesor panameño que dictó la mayoría de los cursos de la disciplina. El mayor peso de la formación de los primeros egresados/as de filosofía recayó sobre él. Domínguez Caballero nació en el 1915 y murió en el 2011. Junto a Ricaurte Soler son las dos figuras de la filosofía en Panamá con mayor reconocimiento a nivel mundial. Su educación superior de posgrado fue realizada entre Estados Unidos y España. Fue un hombre activo hasta los últimos días, dio conferencia, escribió artículos periodísticos y bregó a favor de la filosofía. Como él mismo lo decía, el camino por recorrer es largo, es una carrera de relevo. Sus estudiantes de aquel entonces, hoy profesores del Departamento de Filosofía, dan buen testimonio de él, a pesar de discrepar ideológicamente.

La influencia del pragmatismo norteamericano caló en Domínguez Caballero, lo cual se refleja en sus textos, en donde no anda con rodeos y va directo al punto, no da vueltas especulativas, ni tampoco es pesado leerlo, sus escritos son entendibles sin ninguna pretensión más que enseñar y dejarse entender, es decir, hacer asequible la filosofía al público en general. Por otra parte, Domínguez Caballero siempre estuvo estrictamente preocupado por los problemas filosóficos y su pedagogía nunca se apartó del academicismo, posición que, suponemos, se debe a nuestra tradición que, hasta cierto punto, puede considerarse descontextualizada de los procesos sociales y políticos, con raras excepciones.

Otros factores constantes en su cincelar fue atender y criticar el rol de la universidad en el desarrollo de las actividades concernientes a la filosofía en particular. Naciente compromiso que dio la forma a una tradición, por mínima que sea, y encontró sus mayúsculos resultados en gran pléyade de egresados. Se cuestionó por la esencia de lo panameño y otros estudiantes, con otros enfoques, también se acercaron a esta temática.

Cabe señalar esta consigna, "sin universidad no hay filosofía"; esta tesis fue planteada por el brasileño José Antonio Tobías en la obra $O$ ensino da filosofía das universidades brasileiras, compilación y estudio que había realizado Diego Domínguez, de ese autor y otros tres: Harold A. Durfee y Harold E. Davis en The teaching of philosophy in universities of the United States. Diego Domínguez analizó la obra de José Antonio Tobías, quien señaló que: "Universidad y filosofía 
tienen una existencia conjunta, una no puede existir sin la otra. Es necesario tener un concepto de la Universidad previo a la creación de esta" (DOMíNGUEZ, D., 1968). Así, según ambos autores, queda establecida esa reciprocidad universidad/filosofía que era muy propio del quehacer filosófico de Domínguez Caballero: desarrollar una filosofía académica, pero preocupada por los temas de la sociedad. En el transcurso de su vida profesional hizo sentir sus palpitaciones en todos los rincones académicos de la universidad, como el referente en filosofía, institucionalizó, nacionalizó y organizó el Departamento de Filosofía de la Universidad de Panamá.

Ahora, abordaremos sucintamente los planteamientos de Domínguez Caballero sobre la FE. En la antología que preparó Francisco Céspedes sobre la educación en Panamá hay varios capítulos de sumo interés, entre esos, uno de Domínguez Caballero titulado: "Problemática de una Filosofía de la educación para la escuela panameña", en donde dibuja, a grandes rasgos, lo esencial de la FE en Panamá. Como decíamos, nuestro autor estaba influenciado por el pragmatismo, por ello inicia su reflexión al señalar que, parte de la problemática es no tener un buen planteamiento, sin argüir diatribas dialécticas ni nada por el estilo.

Señala que, en la nomenclatura de FE, hay dos términos que conviene aclarar:

Filosofía y Educación. El Término filosofía se ha de entender como teoría (desfile de ideas como pensaban los griegos). Es decir, una meditación filosofía, basada en investigaciones y observaciones, que nos lleve al encuentro de las cosas que saben hacer y la meta que se debe perseguir. Quiero señalar, en este momento, la desgraciada separación que formular algunos enseñadores entre prácticas y teoría. La historia nos ha probado una y otra vez que nada hay más práctico que la pura teoría. Es la teoría la que nos lleva al desarrollo de una acción lógica y racional [...] en cuanto a la palabra educación... hay que distinguir entre el adiestramiento y educación. No se trata de aprender determinadas habilidades para luego encajar como una pieza armónica en el mecanismo del estado. Esto convertiría al hombre en una especie de tuerca o tornillo en el complicado mecanismo de Leviatán [...] Verdadera educación es la que forma la personalidad del individuo como individuo, que le reconoce sus inalienables derechos. (DOMÍNGUEZ,1985, p. 441-443)

Nuestro autor llama la tención sobre este punto porque suele confundirse una cosa con la otra. Señala que la confusión puede llevar a "oscuros criterios de juicio", muy a menudo, cuando no hay especialistas sobre la materia. En la universidad no se pueden permitir este tipo de libertades, ya que, en teoría, debe 
ser un espacio para la rigurosidad y la erudición en cada ámbito del saber. No toda la educación debe presuponer una reflexión filosófica inmediata, pero sí hay problemas de la educación que son inminentemente filosóficos como, por ejemplo, la concepción del ser humano que debe estar implícita en todo proceso educativo, independientemente del ámbito del saber, así sea técnico. Por una razón sencilla, como señalará Domínguez Caballero, "la posición del conocimiento filosófico no sólo es importante sino es básica y es esencial" (DOMíNGUEZ, 1985, p. 444). Si se prescinde de ella, por muy práctico que se quiera ser, el proceso educativopedagógico quedaría vació, sin contenido.

Nuestra particularidad histórica, nuestra angostura geográfica, determinó nuestra función en el sistema mundo, de tal forma que, de alguna u otra forma tenemos que otear con esa realidad. Muchas veces perdemos la orientación correcta por no tener un plan general trazado. Esa tarea le corresponde - según nuestro autor - a la Universidad de Panamá. A la formación, a la investigación que tiene que emerger del seno universitario.

Domínguez Caballero, le daba un peso tremendo a la FE, y al papel de ésta en la concepción que se tuviese de la propia educación en general. A tal punto que la consideraba como la forjadora de una personalidad y de una nación. En ese sentido, remarcó la importancia de dos puntos a desarrollar: primero, la necesidad de una cultura filosófica en nuestros educadores; y segundo, lo relacionado al Ser y Esencia de lo panameño. Ambos temas se entrecruzan. Es lo que señala el pensador español Carlos Beorlegui al hablar de la búsqueda incesante de identidad del pensamiento filosófico latinoamericano.

\section{Curso}

Oficialmente, la mayoría de los profesionales que imparten cursos de Filosofía, de alguna u otra forma han estado vinculados a la Universidad de Panamá, a menos que hayan estudiado en el extranjero e impartan clases en instituciones privadas. Panamá es un país cerrado, proclive al encapsulamiento académico. Por tener estas características, los dedicados a la filosofía, profesionalmente, están en la comunidad académica alrededor del Departamento de Filosofía de la Universidad de Panamá. Este departamento tiene dos escuelas: una de Ética y Valores y otra de Filosofía e Historia.

Es El Departamento de Filosofía la unidad académica de la Universidad de Panamá la que imparte un curso con el título "Filosofía de la educación", por lo tanto, veremos que se enseña en este curso. Son herramientas para realizar, porteriormente, estudios comparativos regionales si es posible. Este departamento 
ofrece dos licenciaturas: en Humanidades con especialización en filosofía, ética y valores; y humanidades con especialización en filosofía e historia.

Según el plan antiguo de la licenciatura en filosofía, ética y valores, el curso (denominación) se da en IV de la carrera, es decir, el último año; sólo se da un semestre, por lo tanto, son 3 créditos en toda la carrera. En esta misma licenciatura, en el plan nuevo se da un semestre, con la diferencia que es en el primer año. La otra licenciatura que oferta el departamento, de filosofía e historia, no tiene este curso, por lo tanto, si queremos conocer donde encaja, lo podríamos sintetizar con los objetivos y descripción de la primera licenciatura, tanto en su plan antiguo, como el nuevo del 2016.

\begin{tabular}{|l|}
\hline \multicolumn{1}{|c|}{ Plan antiguo } \\
\hline Objetivos: \\
Satisfacer la demanda existente en la sociedad de conocimientos filosóficos \\
fundamentales en el campo de los valores morales y cívicos. \\
Formar recursos humanos con capacidad académica en el campo de la \\
filosofía, la ética y los valores, capaces de desempeñar eficientemente las \\
labores de docencia y asesorías en las áreas administrativas y, políticas y \\
económicas.
\end{tabular}

\section{Plan nuevo (2016)}

Objetivos y descripción:

Esta carrera tiene tres objetivos principales. En primer lugar, busca formar profesionales con una sólida formación filosófica que les permita analizar e interpretar el contexto socio-histórico y cultural, transmitir, promover y difundir el discurso filosófico en los diferentes campos de la vida social, realizar investigación e integrar el saber filosófico en otros campos. En 
segundo lugar, dar al estudiante los conocimientos, marcos de referencia, elementos teóricos y metodológicos necesarios para comprender el desarrollo de las ideas filosóficas y aplicarlas en el análisis y solución de los problemas de la comunidad, de manera que contribuya con el desarrollo material y espiritual a nivel local, regional y nacional. En tercer lugar, generar espacios de reflexión ético-filosófico-políticos que posibiliten la elaboración de estrategias conceptuales y metodológicas genuinas, creativas, integradoras e influyentes para el tratamiento de los problemas más acuciantes a los que se enfrenta la sociedad panameña. En ese sentido, el egresado de esta carrera posee una actitud crítico-analítica de los problemas del hombre y de la unificación e integración del conocimiento.

Ahora veremos el contenido del curso de FE, propiamente. Los contenidos, para tener una idea de lo que se enseña en este curso. Un tema a considerar es que en la Universidad de Panamá no está muy bien establecido el sistema de cátedra, pero si es habitual que los profesores den cursos por áreas de afinidad. Lo cual es una limitante, ya que no hay una tradición, salvo raras excepciones, de concebir un curso bajo ideal de la cátedra. Hay cierta regularidad, pero los cursos varían de profesor/a frecuentemente.

\section{Asignatura: Filosofía de la Educación}

Objetivos generales: referidos a los aspectos globales que en el campo de la teoría y de las actitudes, se esperan obtener de los estudiantes.

Objetivos específicos: Referidos a las habilidades, destrezas y capacidad de efectuar operaciones, que atreves del curso desarrollará el estudiante.

\section{Contenidos:}

Incluye temas y sub-temas que de acuerdo a los objetivos se desarrollaran para lograr lo propuesto:

1. ¿Qué es la filosofía? (Antropología, Cultura, Educación)

1.1. El origen de la filosofía

1.2. Concepto de Filosofía

1.3. Dominio de la Filosofía: Tema y Disciplina

1.4. Esencia de la Filosofía

1.5. Métodos de la Filosofía

1.6. Proceso histórico de la Filosofía

2. ¿Qué es educación? (Antropología, Cultura, Educación)

2.1. El Ser del hombre

2.2. La Cosmovisión básica (Idealismo, Realismo etc....)

2.3. La educación: proceso o resultado

2.4. Concepto de educación

2.5. Cambios básicos en la educación

2.6. Educación y transcendencia 


3. ¿Qué es Filosofía de la educación? (Individualismo, Nacionalismo,
Culturalismo)
3.1. Naturaleza y alcance de la Filosofía de la educación
3.2. Función de la Filosofía de la educación
3.3. La Filosofía de la educación como disciplina autónoma
3.4. La función crítica de la Filosofía de la educación
3.5. Estado actual de la Filosofía de la educación
3.6. Definiciones de Filosofía de la educación crítica
4as filosofías fundamentales y los movimientos educativos actuales
4.1. La Filosofía idealista de la educación
4.2. La Filosofía realista de la educación
4.3. La Filosofía pragmática de la educación
4.4. La Filosofía Existencialista de la Educación
4.5. La Filosofía marxista de la educación
4.6. La Filosofía de la democracia
Metodología:
Breve exposición de a metodología a utilizar por el profesor, por ejemplo:
conferencias, debates, mesas redondas, laboratorios, incluyendo el
repertorio de métodos y técnicas que utilizará también el estudiante.

\section{A modo de conclusión}

Diego Domínguez Caballero es la figura pivote de la FE en Panamá. Fijó su atención en el carácter central que debe jugar la filosofía en la universidad y ésta en la sociedad; además de enfatizar y desarrollar un trabajo que se indagara por el Ser panameño para darle una identidad, tanto al individuo, como a la nación. Por nuestra cuenta, reconocemos la importancia de las reflexiones y preocupaciones de Domínguez Caballero, pero no las suscribiríamos totalmente en la actualidad, más que todo, por el esencialismo implícito en su propuesta.

Por otra parte, sin duda alguna se tendrá que actualizar el programa de FE. Hay mucha bibliografía científica al respecto. Además, urge una historia de la FE en Panamá, que sistematice todo lo que ésta signifique para un contexto como el nuestro. Esto es uno de los principales problemas que se desprenden cuando no hay cátedras especializadas, es decir, un profesor o profesora a dedicación de tiemplo completo sobre la temática en particular, que produzca en función de su especialidad. Y eso no sólo ocurre con FE, sino con casi todos los otros cursos, con raras excepciones. Eso es el resultado, entre cosas, de la escolarización de la universidad, es decir, la sobrevaloración del papel del profesor en el aula, con lo que se descuidan, totalmente, la investigación científica, cuando ambas cosas deberían ir de la mano.

Con ese escenario tenemos tareas pendientes. En los tres niveles que hemos enfocado nuestra brevísima reflexión, hay mucha tela por cortar. Hoy, 
Diego Domínguez Caballero como gran parte de nuestras tradiciones de pensadores y pensadoras está en el museo de la biblioteca; no se cuenta con una producción científica en filosofía de la educación para los efectos del curso y no contamos con una historia general de la educación en Panamá en términos teóricos; el referente es el texto de Francisco Céspedes citado. Sirvan estas líneas como una introducción e incentivo para emprender las tareas pendientes.

\section{REFERÊNCIAS}

CANTON, A. Hacia una nueva escuela rural americana. Panamá: s.e,1952.

CÉSPEDES, F. La educación en Panamá: panorama histórico y ontología. Panamá: Biblioteca de la Cultura Panameña, 1985.

DE LA ROSA, D. “Educación humanista o educación pragmática” en: CÉSPEDES, F. La educación en Panamá: panorama histórico y ontología. Panamá: Biblioteca de la Cultura Panameña, 1985.

DEL VASto, C. Universidad de Panamá. Origenes y Evolución. Panamá: Universidad de Panamá, 2010.

Dolores Moscote, J. \& MÉNDEZ PEREIRA, O. Itinerario. Selección de discursos, ensayors y conferencias / Historia de la instrucción pública en Panamá. (Prólogo Pedro Pineda González), Panamá: Autoridad del Canal de Panamá, 1999.

DomíngueZ, D. Los Estudios Filosóficos en la Universidad de Panamá. Panamá: Universidad de Panamá, 1963.

DomíngueZ, D. Examen Crítico de la Enseñanza de la Filosofía en América. Panamá: Universidad de Panamá, 1968.

DOMínGUEZ, D.“Esencia y problemática del hombre actual (I)”. Revista Lotería. No.208, 16,140, 1973.

DomínguEZ, D. "Servicio a la Universidad de Panamá". Revista Lotería. No. 263, pp, 1$15,1978$.

DOMíNGUEZ, D."Problemática de una filosofía de la educación para la escuela panameña" en: CÉSPEDES, F. La educación en Panamá: panorama histórico y ontología. Panamá: Biblioteca de la Cultura Panameña. pp. 441-447, 1985.

DOMínGUEZ, D. “En la búsqueda de lo panameño”. Revista Lotería. No.385, pp, 216-223, 1991.

DomínGUEZ, D. Razón y Sentido de lo panameño. Ensayo. Panamá: Universidad de Panamá, 2004. 
MORENO, DAVIS, J, C. Historia, espíritu y autenticidad de la filosofía en Panamá y panameña. Panamá: IDEN, 2006.

PORCELL, N.Los Docentes Europeos y la Formación de la Universidad de Panamá. Panamá: Universidad de Panamá: ICASE, 1991.

PRADO, S. Educación liberal y educación conservadora en Panamá: una aproximación crítica (1903-1953). (Tesis de Maestría). Panamá: UDELAS, 2010.

PRADO, S. "Educación Liberal y Educación Conservadora en Panamá: una aproximación crítica (1903 - 1953)”. Revista Filosofía y Sociedad. No.1, 77-86, 2011.

RITTER, L, P. Filosofía de la nación romántica (Seis ensayos críticos sobre el pensamiento intelectual y filosófico en Panamá 1930 - 1960). Panamá: Editorial Mariano Arosemena, 2007.

RodríGuez ReYES, A. "Diego Domínguez Caballero y algunas cuestiones nucleares de la filosofía en Panamá”. Ensayos Pedagógicos, VII(1), pp. 15-31, 2012.

ROMERO, P. "En torno al aprendizaje reflexivo", en: La Estrella de Panamá (11.11.2016), 2016. 